\title{
The Comparison of Ultrasound and Fluoroscopy- guided for Determining Puncture Entry Point of Percutaneous Vertebroplasty
}

\section{Sen Ye ( $\sim$ yesenspine@sina.cn )}

The First Affiliated Hospital of Chongqing Medical University https://orcid.org/0000-0003-3864-367X

Xiaorong Lai

The First Affiliated Hospital of Chongqing Medical University

Lingjian Zhong

The First Affiliated Hospital of Chongqing Medical University

Changzhang Yu

The First Affiliated Hospital of Chongqing Medical University

\section{Yanzhen Chen}

The First Affiliated Hospital of Chongqing Medical University

\section{Research}

Keywords: Ultrasound-guided, fluoroscopy-guided, puncture entry point percutaneous vertebroplasty, osteoporotic vertebral compression fracture

Posted Date: January 19th, 2021

DOI: https://doi.org/10.21203/rs.3.rs-149179/v1

License: (c) (i) This work is licensed under a Creative Commons Attribution 4.0 International License. Read Full License 


\title{
The comparison of ultrasound and fluoroscopy-guided for
}

\section{determining puncture entry point of percutaneous}

\section{vertebroplasty}

Author information:Sen Ye,Xiaorong Lai,Lingjian Zhong,Changzhang Yu and Yanzhen Chen.

First author: Sen Ye. E-mail:yesenspine@sina.cn.

Corresponding author: Yanzhen Chen. E-mail: 13507071872@163.com.

Affiliation: Department of Spine Surgery, Xingguo County People's Hospital, Ganzhou, Jiangxi.

\begin{abstract}
OBJECTIVE: The purpose of this study was to compare the safety and efficacy of ultrasound and fluoroscopy-guided for determining the puncture entry point of percutaneous vertebroplasty (PVP).

Methods: From November 2018 to December 2019, a total of 50 eligible patients with thoracolumbar osteoporotic vertebral compression fractures (OVCFs) treated by PVP were retrospectively collected, of which 25 cases were in ultrasound-guided group and the other 25 cases were in fluoroscopy-guided group. Radiation doses, one-time localization success rate, operation time, clinical efficacy and complications incidence were compared between the two groups.
\end{abstract}

Results: Ultimately, 46 patients completed followed-up, whose average follow-up time was 9 months, the dose and times of fluoroscopy and operation time in ultrasound-guided group statically significantly decreased compared with fluoroscopy-guided group $(\mathrm{P}<0.01)$. The one-time localization success rate in ultrasound-guided group and fluoroscopy-guided group were $68.2 \%$ and $37.5 \%$ respectively $\left(x^{2}=4.33, \mathrm{P}=0.037\right)$, whose difference was statistically significant $(\mathrm{P}<0.01)$. However, there was no significant difference in the visual analog scale (VAS) scores of back pain relief between the two groups $(\mathrm{P}>0.05)$. There were no postoperative complications such as infection, hematoma formation and fracture nonunion observed between the two groups.

Conclusion: Ultrasound-guided entry point determination for PVP puncture is safe, which can significantly improve the success rate of one-time localization and decrease radiation exposure and operation time.

Key words: Ultrasound-guided; fluoroscopy-guided; puncture entry point percutaneous vertebroplasty; osteoporotic vertebral compression fracture

\section{Introduction}

Osteoporotic vertebral compression fracture (OVCFs) is one of the most serious complications of osteoporosis (OP). At present, PVP is one of the effective treatment methods for OVCF, which is highly recognized for its advantages such as less trauma, 
significant pain relief and fast functional recovery[1-3]. The percutaneous transpedicular puncture is the first step to perform PVP procedure, which is usually guided by X-ray fluoroscopy. However, frequent X-ray fluoroscopy will lead to the increase of radiation exposure to both surgeons and patients[4]. Ultrasonography (US) has been widely used in clinical practice[5]. US could real-time monitor the puncture procedure without ionizing radiation, since it could distinguish bones, nerves, blood vessels and muscles clearly. Ultrasound scanning technology has been gradually applied in orthopedics, such as lumbar facet joint injection and nerve root block[6, 7]. Up to date, the safety and efficacy of ultrasound guidance for determining the entry point of PVP puncture has not been reported yet.

The aim of the present retrospective controlled study was to introduce US to determine the puncture entry point to reduce the radiation exposure and puncture time during PVP procedure.

\section{Materials and Methods}

\section{Patients}

Inclusion criteria were as follows: 1) age $\geq 55$ years, T10-L2 OVCFs; 2) vertebral column height loss $\geq 15 \%$; 3) moderate and severe pain caused by fractures in the chest and waist (VAS scores $\geq 4$ ), conservative treatment can not relieve the pain; 4) back pain for $\leq 6$ weeks; 5 ) bone mineral density (BMD) score less than $-2.5 ; 6$ ) MRI SRIT sequence shows high signal of vertebral body.

Exclusion criteria were as follows:1) other pathological vertebral fractures, such as multiple myeloma, bone metastases, giant cell tumor of bone and hemangioma; 2) the posterior edge of the fractured vertebral body protrudes into the spinal canal and obviously oppresses the dural sac; 3) The compression degree of the vertebral body exceeds $75 \%$; 4) multiple vertebral fractures; 5) with symptoms of nerve injury; 6) blood coagulation disorders; 7) with severe medical diseases or mental illness, not suitable for surgery.

A total of 50 patients with OVCFs were enrolled, including 16 males and 34 females, aged from 63 to 78 years. There were 25 cases in ultrasound-guided PVP group and 25 cases in traditional X-ray guided PVP group. In the ultrasound-guided PVP group, there were 8 males and 17 females, the average age was $69.5 \pm 3.9$ years (63-77 years) and the time from fracture to operation was $2.9 \pm 0.8$ days (2-4 days). In the traditional X-ray guided PVP group, there were 8 males and 17 females, the average age was $69.6 \pm 5.4$ years(61-79 years), and the time from fracture to operation was $2.9 \pm 1.3$ days (1-5 days). There was no significant difference in sex, age, time from fracture to operation, BMD, BMI and preoperative VAS score between the two groups (Table 1). The ethics committee of our hospital approved the present study (2018-06).

\section{Operative Technique}

Ultrasound-guided PVP group: the patient took the prone position and used a curved low frequency $(2-5 \mathrm{MHz})$ probe to make the image field of vision which is enough to contain 2 vertebrae for observation. The ultrasonic images of spinous 
process, articular process, transverse process, vertical spinal muscle, psoas muscle and psoas major muscle were monitored when the ultrasonic probe was scanning at the midline of the spine. The direction of the probe was gently adjusted toward the head or foot.

When the midline $3-5 \mathrm{~cm}$ was opened beside the spinous process, a parasagittal scan showed an intermittent hyperechoic wave curve, that was the transverse process. The vertical spinal muscle was in the superficial layer of the transverse process, and the psoas major muscle was between the adjacent transverse process and its deep layer. This imaging was described as a "Trident" sign (Fig.1, Fig.2).

By slowly translating the probe to the inside, A continuous wavy high echo line emerged, which was the imaging of the facet joint, and the highest point of the wave was the intersection of the superior articular process and the inferior articular process. When slightly outward translation of the probe, the image appeared to be half of the Chinese character "convex", this was the image of the facet joint and transverse process. When scanning the transverse process in the axial direction, the connecting part between the superior articular process and the transverse process could be seen at the same time, and the probe moved slightly to the foot on the basis of the horizontal cross section of the transverse process. When the convex part of the superior articular process disappeared, the "inverted triangle"-shaped soft tissue acoustic image below it could be seen, which was the vertex of the herringbone ridge and the puncture location (Fig.3, Fig.4).

PVP was performed using fluoroscopy guidance with bilateral transpedicular injection of bone cement (poly-methylmethacrylate; PMMA) after determining the needle entry point. The radiation doses of the patient were collected during the operation.

Traditional X-ray-guided PVP group: PVP was completely performed under the fluoroscopy guidance to determine puncture point.

\section{Outcome Evaluation and Statistical Analyses}

Statistical analysis was performed by SPSS23.0. The patient characteristics were summarized using descriptive statistics and were presented as Mean \pm SD and percentages for continuous and categorical variables, respectively. Differences between groups were assessed using the Student t test and the $x^{2}$ test for continuous and categorical variables, respectively. $\mathrm{P}<0.05$ was considered statistically significant. The radiation doses, X-ray fluoroscopy duration, operation duration, the VAS score ( back pain before operation), the VAS score (back pain 1 day after operation), one-time localization success rate and t complications incidence were statistically analyzed between the two groups.

\section{Results}

Among the 50 patients, 46 patients were followed up. Three patients in ultrasound-guided PVP group lost follow-up, and one patient lost follow-up in traditional X-ray guided PVP group. The average follow-up time was 9 (4-12) months. There were no postoperative complications such as infection, hematoma formation 
and fracture nonunion between the two groups. The one-time localization success in ultrasound-guided PVP group and traditional X-ray-guided PVP group were $68.2 \%$ and $37.5 \%$ respectively $(\mathrm{x} 2=4.33, \mathrm{P}=0.037)$ (Table 2$)$. The dose and the number of fluoroscopy and operation time in ultrasound-guided PVP group significantly decreased compared with traditional $\mathrm{X}$-ray guided PVP group $(\mathrm{P}<0.01)$. There was no significant difference in the VAS score of back pain relief between the two groups (Table 3).

\section{Discussion}

C-arm fluoroscopy has been used to adjust the puncture entry point during traditional PVP procedure. The fluoroscopy-guided localization of puncture point is not accurate enough resulting in excessive radiation exposure[8]. This study is the first time to introduce ultrasound guidance to determine the puncture entry point of PVP, which demonstrated it significantly reduced dose and fluoroscopy times than those with traditional X-ray guidance.

Previous studies have shown that $90 \%$ of the effective radiation dose received by orthopedic surgery comes from PVP procedure. More and more evidence has proved that high-dose ionizing radiation can directly cause many diseases, which seriously affect both surgeon and patents' health[9]. Several measures have been reported to decrease the radiation exposure, like using mobile lead shield, wearing lead collars, and whole body lead aprons, changing the position of X-ray source[10-12]. However, even low-dose radiation has been correlated with radiation-related disease development[13]. Thus, the importance to further reducing radiation exposure can never be emphasized too much. In the present study, ultrasound was used to determine the insertion point, which could provide real-time and high-resolution images without any radiation. Our results showed that the times of fluoroscopy in ultrasound guidance group was $50.2 \%$ lower than those in traditional X-ray guidance group, leading to $8 \%$ lower in radiation dosage.

Furthermore, shorter operation time was also observed in ultrasound guidance group. It was quite time-consuming to frequently use C-arm during PVP procedure due to the switch between AP and lateral fluoroscopy. On the other hand, ultrasound could provide real-time and high-resolution image to help surgeon locate puncture entry point continuously and accurately, which saved a lot of time. On account of local anesthesia for PVP treatment, less operation time can help patients more tolerant to operative procedure, especially for the senile.

In recent years, some novel ultrasound navigation technologies have been used in PVP surgery. Xu, et al[14] have reported a "Targeted Percutaneous Vertebroplasty" that is a $3 \mathrm{D}$ model of fractured vertebra based on preoperative CT scanning data is built preoperatively, and with the help of preoperative digital design, bilateral skin entry points, needle trajectory, and needle insertion depth are established. However, preoperative CT scanning is not exactly the same posture as that in the real operative procedure, whose deviation could result in serious errors for puncture complications. Another study[13] used ultrasound volume navigation (UVN) to reduce the radiation exposure and puncture time of percutaneous transpedicular puncture in PVP and PKP, which is a new navigation technique, can remedy the limitations of each imaging 
modality by fusing real-time US and preoperative MRI or CT data. However, spine surgeons might be not familiar with it.

In conclusion, the determination of puncture entry point under the ultrasound guidance is safe that can improve the success rate of one-time localization, and further reduce the radiation dose and the number of X-ray fluoroscopy in PVP. However, the number of cases included in this study is relatively small, and a larger sample of clinical studies is needed. Furthermore, spine surgeons should undergone comprehensive medical training about ultrasound technique before performing spine surgery.

\section{Declarations}

\section{Abbreviations}

PVP: percutaneous vertebroplasty; OVCFs: osteoporotic vertebral compression fractures;VAS: visual analog scale;OP: osteoporosis; US:ultrasonography; BMD: bone mineral density; PMMA: poly-methylmethacrylate; UVN: ultrasound volume navigation.

\section{Acknowledgements}

We would like to thank all the participating Trauma Medical Directors who shared their time, experience, and protocol information for this survey

\section{Authors' contributions}

S Y and YZ C participated in conception and design of this study. SY and XR L performed the acquisition of data. LJ Z and CZ Y performed the statistical analyses. $S$ Y drafted the manuscript. YZ Crevised the manuscript for important intellectual content. All authors read and approved the final manuscript.

\section{Funding}

This study is funded by Foundation of JiangXi Science and Technology Department(20181BBG78020).

\section{Availability of data and materials}

All data generated or analyzed during this study are included in this published article.

\section{Ethics approval and consent to participate}

This study was approved by the ethics committee of Xingguo County People's Hospital (No.2018-06). The study was approved with a partial waiver of consent, waiving the requirement for a conform containing a signature of the participant.

\section{Consent for publication}

Not applicable

\section{Competing interests}

The authors declare that they have no competing interests.

\section{Reference}

1. Xie, L., et al., Percutaneous vertebroplasty versus conservative treatment for osteoporotic vertebral compression fractures: An updated meta-analysis of prospective randomized controlled trials. Int J Surg, 2017. 47: p. 25-32.

2. Wencheng Yang, J.S., Ming Liang, Hao Cui, Hengyi Chen, Jianyi Yang, Functional Outcomes and New Vertebral Fractures in Percutaneous Vertebroplasty and Conservative Treatment of Acute Symptomatic Osteoporotic Vertebral Compression Fractures. World Neurosurgery, 2019. 
3. Yang, E.Z., et al., Percutaneous Vertebroplasty Versus Conservative Treatment in Aged Patients With Acute Osteoporotic Vertebral Compression Fractures: A Prospective Randomized Controlled Clinical Study. Spine (Phila Pa 1976), 2016. 41(8): p. 653-60.

4. Zhang, L., et al., Unipedicular versus bipedicular percutaneous vertebroplasty for osteoporotic vertebral compression fractures: a prospective randomized study. BMC Musculoskelet Disord, 2015. 16: p. 145.

5. Apard, T., Ultrasonography for the orthopaedic surgeon. Orthop Traumatol Surg Res, 2019. 105(1S): p. S7-S14.

6. Takeuchi M, K.M., Wakao N, et al, A Simple, 10-minute Procedure for Transforaminal Injection under Ultrasonic Guidance to Effect Cervical Selective Nerve Root Block. Neurol Med Chir (Tokyo) 2014.

7. Ling Ye1, C.W.a.H.L., Ultrasound-guided versus low dose computed tomography scanning guidance for lumbar facet joint injections: same accuracy and efficiency. BMC Anesthesiology 2018.

8. Cannavale, A., et al., Percutaneous vertebroplasty with the rotational fluoroscopy imaging technique. Skeletal Radiol, 2014. 43(11): p. 1529-36.

9. von Wrangel, A., A. Cederblad, and M. Rodriguez-Catarino, Fluoroscopically guided percutaneous vertebroplasty: assessment of radiation doses and implementation of procedural routines to reduce operator exposure. Acta Radiol, 2009. 50(5): p. 490-6.

10. HARSTALL R, H.P.F., MINI R L, et al, Radiation Exposure to the Surgeon DuringFluoroscopically Assisted Percutaneous VertebroplastyA Prospective Study. Spine (Phila Pa 1976), 2005. 30(16).

11. FITOUSI N T, E.E.P., DELIS H B, et al, Patient and staff dosimetry in vertebroplasty. Spine (Phila Pa 1976), 2006. 31(23).

12. C, C.H., Fluoroscopic Radiation Exposure during Percutaneous Kyphoplasty. J Korean Neurosurg Soc, 2011. 49(1).

13. Zhao, Y., et al., Reduced Radiation Exposure and Puncture Time of Percutaneous Transpedicular Puncture with Real-Time Ultrasound Volume Navigation. World Neurosurgery, 2018. 119: p. e997-e1005.

14. Xu, J., et al., "Targeted Percutaneous Vertebroplasty" Versus Traditional Percutaneous Vertebroplasty for Osteoporotic Vertebral Compression Fracture. Surgical Innovation, 2019. 26(5): p. 551-559. 
Figures

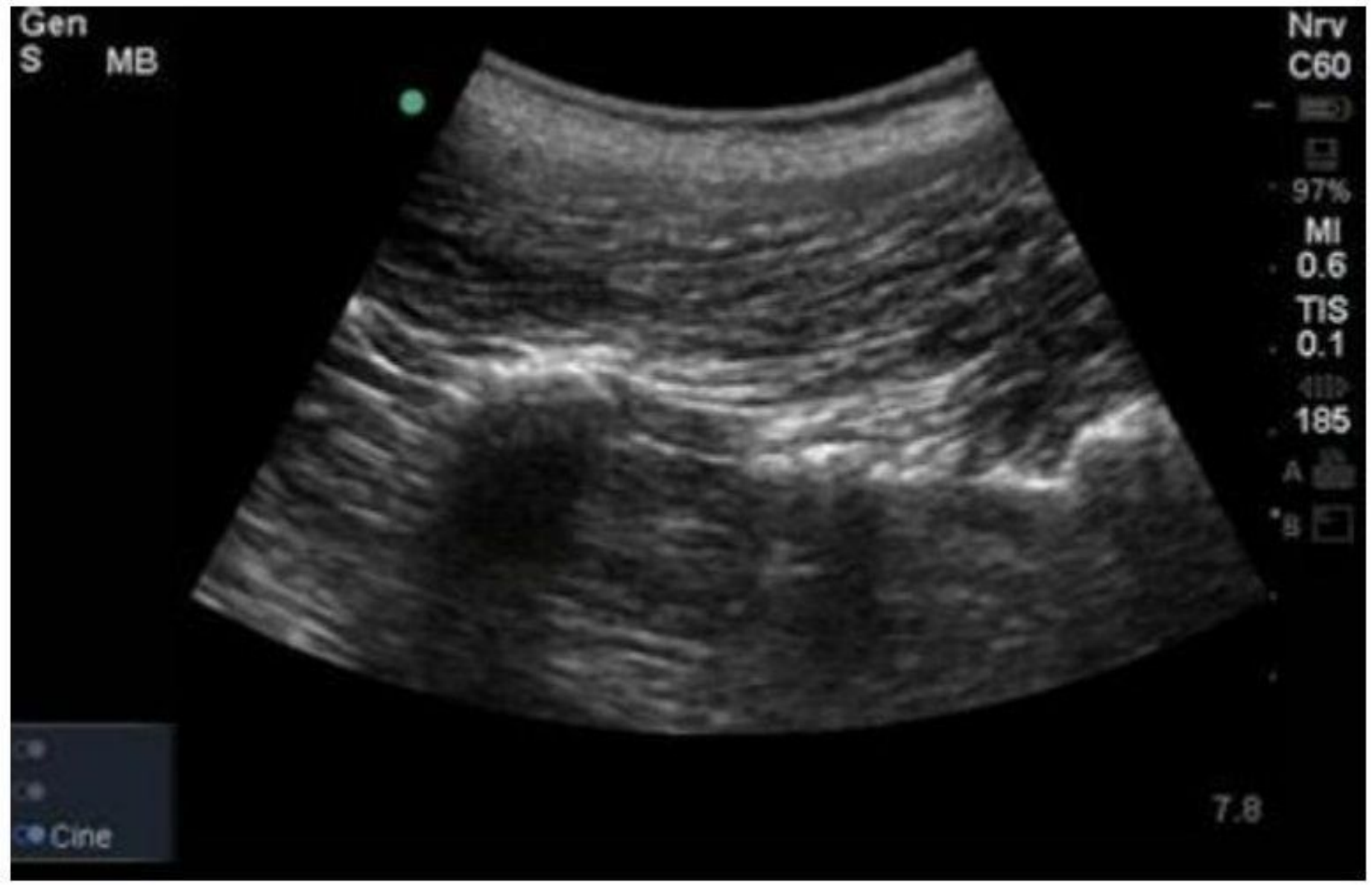

Figure 1

Sagittal section of transverse process 


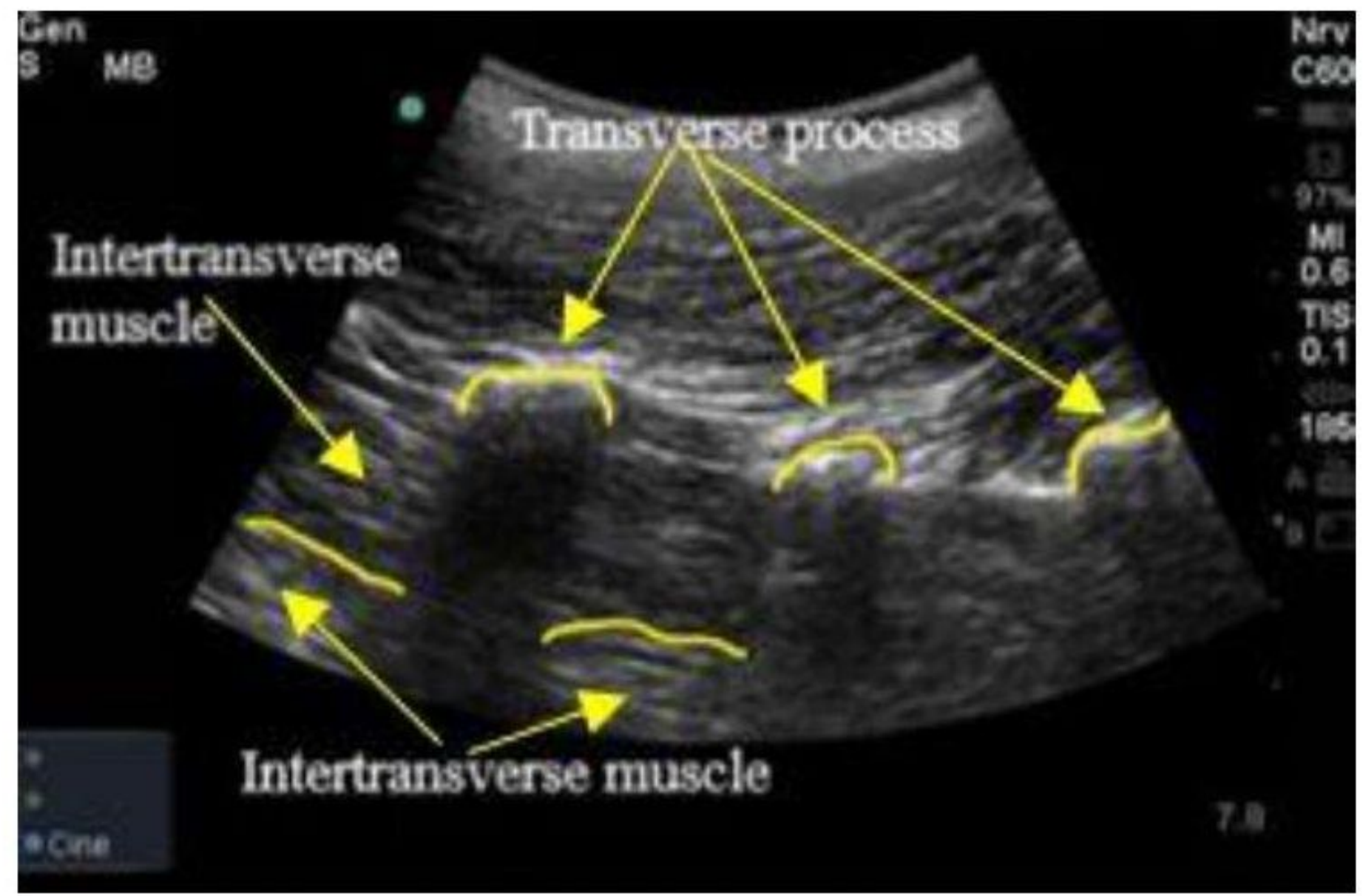

Figure 2

That was the vertical spinal muscle, transverse process, intertransverse process and the psoas major muscle. This imaging was described as a "Trident" sign $\rrbracket$ 


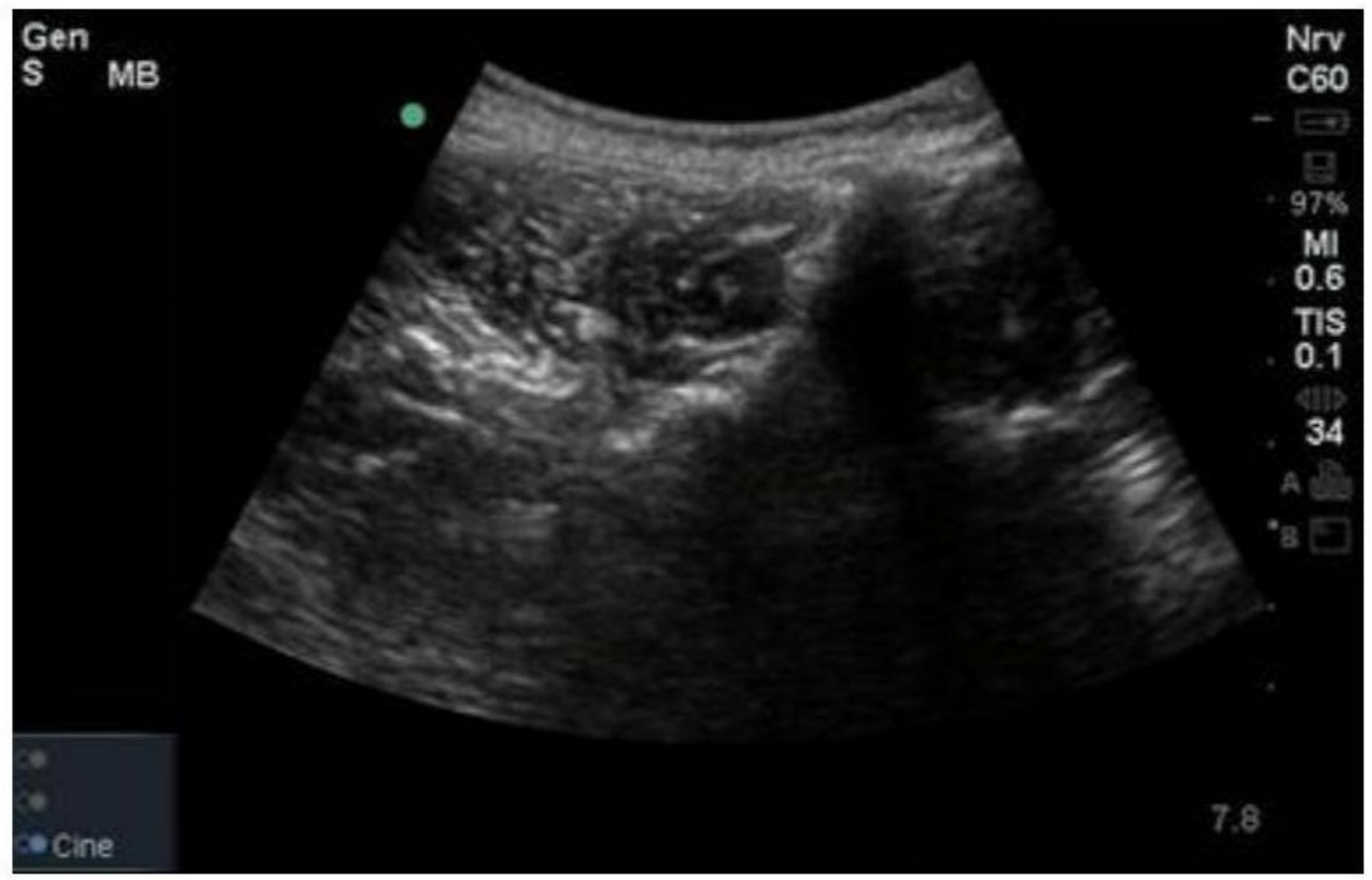

Figure 3

Cross section of herringbone crest 


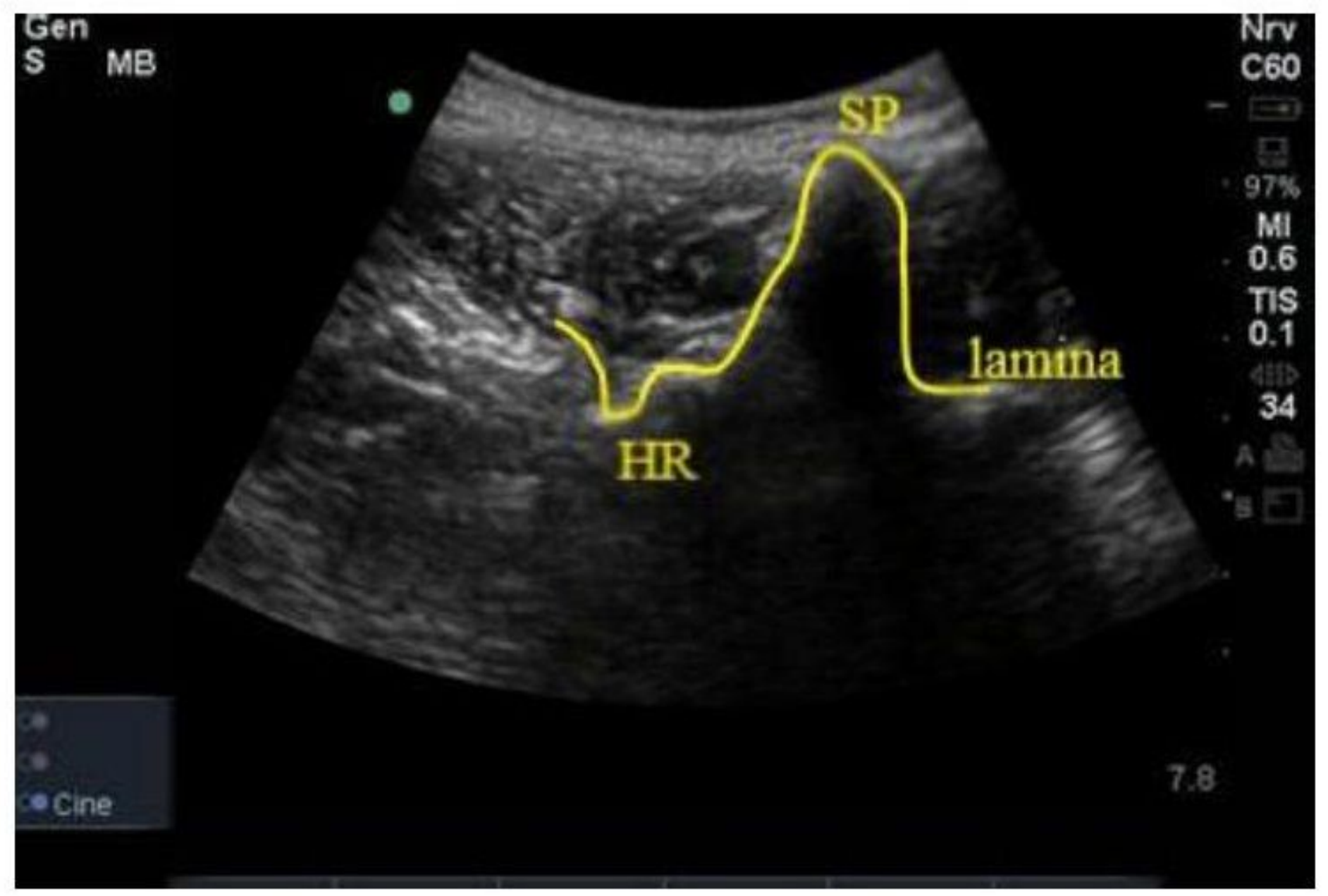

\section{Figure 4}

When the convex part of the superior articular process disappeared, the "inverted triangle"-shaped soft tissue acoustic image below it could be seen, which was the vertex of the herringbone ridge and the puncture location. Spinous process(SP), herringbone ridge $(\mathrm{HR}) \mathbb{}$

\section{Supplementary Files}

This is a list of supplementary files associated with this preprint. Click to download.

- Table.pdf 